\title{
Thermocapillary Migration of Deformable Bubbles at Moderate to Large Marangoni Number in Microgravity
}

\author{
Jian-Fu Zhao • Zhen-Dong Li • Hui-Xiong Li · Jing Li
}

Received: 28 November 2009 / Accepted: 3 April 2010 / Published online: 22 April 2010

(C) Springer Science+Business Media B.V. 2010

\begin{abstract}
Using the level-set method and the continuum interface model, the axisymmetric thermocapillary migration of gas bubbles in an immiscible bulk liquid with a temperature gradient at moderate to large Marangoni number is simulated numerically. Constant material properties of the two phases are assumed. Steady state of the motion can always be reached. The terminal migration velocity decreases monotonously with the increase of the Marangoni number due to the wrapping of isotherms around the front surface of the bubble. Good agreements with space experimental data and previous theoretical and numerical studies in the literature are evident. Slight deformation of bubble is observed, but no distinct influence on the motion occurs. It is also found that the influence of the convective transport of heat inside bubbles cannot be neglected at finite Marangoni number, while the influence of the convective transport of momentum inside bubbles may be actually negligible.
\end{abstract}

Keywords Thermocapillary migration $\cdot$ Bubble • Level-set method

J.-F. Zhao $(\bowtie) \cdot$ Z.-D. Li · J. Li

Key Laboratory of Microgravity

(National Microgravity Laboratory), Institute of Mechanics,

Chinese Academy of Sciences, Beijing 100190, China

e-mail: jfzhao@imech.ac.cn

H.-X. Li

State Key Laboratory of Multiphase Flow in Power

Engineering, School of Energy and Power Engineering,

Xi'an Jiaotong University, Xi'an 710049, China

\section{Introduction}

A fluid particle (gas bubble or liquid drop) floating in an immiscible bulk fluid with a temperature gradient can be moved by the non-uniform surface tension at the particle interface. This motion is well known as the thermocapillary or Marangoni migration. It plays an important role in many natural physical processes as well as a host of industrial activities, particularly in space material processing and many other scientific and engineering applications in microgravity. Thus, it attracts much more interests of researchers all over the world along with the progress of human space activities. However, most of the work on this subject is relatively recent, as summarized in the monograph by Subramanian and Balasubramaniam (2001). Focusing upon the present topic, only studies relating to the thermocapillary migration of gas bubble are briefly reviewed here.

Thermocapillary migration was first analyzed by Young et al. (1959) in the case of infinitesimal Reynolds and Marangoni numbers, in which convective transport of momentum and heat can be neglected comparing to molecular transport of these quantities and the governing equations can then be linearized. They derived the named YGB theory predicting the following steady migration velocity

$V_{Y G B}=\frac{2 U}{\left(2+3 \mu_{2} / \mu_{1}\right)\left(2+k_{2} / k_{1}\right)}$

where $U=-\sigma_{T} \nabla T_{\infty} R / \mu_{1}$ is the named thermocapillary velocity, $R$ is the bubble radius, $\mu$ is the dynamic viscosity, $k$ is the thermal conductivity, $\sigma_{T}$ is the 
rate of change of interfacial tension with temperature, $\nabla T_{\infty}$ is the temperature gradient imposed in the continuous bulk fluid. The subscripts 1 and 2 denote the material properties of the continuous bulk fluid and the fluid particle, respectively. The Reynolds and Marangoni numbers are defined as $R e=U R / \nu_{1}$ and $M a=U R / \lambda_{1}$. Here, $v$ denotes the kinematic viscosity, $\lambda$ denotes the thermal diffusivity. If the Prandtl number is defined as $\operatorname{Pr}=v_{1} / \lambda_{1}$, one can obtain $M a=\operatorname{Pr} R e$.

The analysis of Young et al. (1959) was extended by many others to include convective influence. For example, Bratukhin (1975) used a regular perturbation expansion in which the Reynolds number was used as a perturbation parameter. He determined the migration velocity correction at $O(R e)$ is zero. Thompson et al. (1980) extended this expansion to the next higher order term and determined a nonzero correction to the migration velocity at $O\left(R e^{2}\right)$. Using the method of matched asymptotic expansions, Subramanian (1981, 1983) provided a correction to the migration velocity, in which the Marangoni number was used as a perturbation parameter. It was shown to be useful only for $M a<0.5$. An extension of Subramanian's results to $O\left(M a^{3}\right)$ by Merritt (1988) was shown to be useful for $M a<2$, while Shankar and Subramanian (1988) constructed an improved series that was useful up to $M a \leq 25$. Crespo et al. (1998) obtained an asymptotic analysis for small Marangoni number case when the velocity field in continuous bulk phase is given by potential flow, which implies large Reynolds number and small Prandtl number in that case. For large Marangoni number case, Crespo and Jimenez-Fernandez (1992a, b) and Balasubramaniam and Subramanian (1996) provided asymptotic analyses for the thermocapillary migration of spherical gas bubbles in both small and large Reynolds number limits.

On the other hand, Shankar and Subramanian (1988), as well as Oliver and De Witt (1994), studied numerically the thermocapillary motion of gas bubbles at zero Reynolds number and Marangoni number as high as 200. They employed the finite difference method to solve the energy equation, but used analytical results for the velocity field. Thermocapillary migration of gas bubbles at moderate to large Reynolds number was also studied numerically by Szymczyk and Siekmann (1988), Balasubramaniam and Lavery (1989), Chen and Lee (1992), Nas and Tryggvason (1993), Welch (1998), and Ma (1998) for the Marangoni number as high as 1,000 . The results are qualitatively consistent with the asymptotic predictions of Balasubramaniam and Subramanian Balasubramaniam and Subramanian (1996). Undeformable bubbles, however, was usually assumed in most studies except Welch (1998), in which a finite volume method with interface tracking capability on a moving unstructured mesh was used to address the effect of bubble deformation on the transient thermocapillary migration in microgravity.

In addition to theoretical and numerical developments, there are some results from experiments in earth-based laboratories, as well as in reduced gravity conditions. Because of the non-linearity of the problem, the thermocapillary motion with finite values of the Reynolds and the Marangoni numbers can be observed experimentally only in microgravity environment in order to avoid the buoyant convection. Hadland et al. (1999) and Kang et al. (2008) reported some experimental results on the thermocapillary migration of air bubbles in silicone oil aboard a NASA space shuttle and the 22nd Chinese recoverable satellite RS-22, respectively, both two experiments cover a wide range of the Reynolds and Marangoni numbers. Some groundbased experiments, for example, Treuner et al. (1996), were also carried. The blend of transients caused both by the short-term microgravity environment utilized and by the thermocapillary migration itself makes their observations fraught with uncertainty.

In the present paper, a numerical study on the thermocapillary motion of deformable bubbles at moderate to large Marangoni number is reported. The results of the thermocapillary migration velocity will be compared with experimental data of Hadland et al. (1999) and Kang et al. (2008), as well as some asymptotic predictions and previous numerical simulations. The structures of the flow and temperature fields will be presented to help understanding the characteristic of this phenomenon.

\section{Mathematical Formulation and Numerical Method}

The thermocapillary migration of a single deformable bubble in an immiscible bulk liquid is considered here with the following assumptions: (1) the fluids in both phases are Newtonian, viscous and incompressible; (2) the material properties are constant and not influenced by the temperature; (3) the surface tension depends linearly upon the temperature; (4) the motion in both phases is axisymmetric and laminar.

To capture the interface of the bubble, the level-set method (Osher and Sethian 1988) is used. The level-set function is denoted as $\varphi$ which is positive in the continuous bulk fluid and negative inside the bubble. So the interface between two phases is the zero level-set of 
$\varphi$, which can be advanced by the following convection equation:

$\frac{\partial \varphi}{\partial t}+\boldsymbol{u} \cdot \nabla \varphi=0$

where $\boldsymbol{u}$ denotes the velocity vector. A third-order Runge-Kutta method in time and a fifth-order WENO method in space are used to solve the equation.

In the numerical simulation, however, $\varphi$ will no longer be a distance function as the interface evolves. In order to keep $\varphi$ approximately equal to signed distance, the re-initialization equation (Sussman et al. 1994)

$\frac{\partial \varphi}{\partial \tau}=\operatorname{sgn}\left(\varphi_{0}\right)(1-|\nabla \varphi|)$

needs to be solved to steady state periodically during simulation with the following initial condition

$\varphi(\boldsymbol{x}, 0)=\varphi_{0}(\boldsymbol{x})$

where $\tau$ is the virtual time, $\varphi_{0}$ is the level-set function at the time $t$ and $\operatorname{sgn}()$ is the smoothed sign function.

Furthermore, to guarantee the mass conservation of the bubble the following Hamilton-Jacobi equation is also solved to steady state periodically during simulation (Son and Dhir 2007)

$\frac{\partial \varphi}{\partial \tau}+\left(V_{0}-V\right)\left(a+b \kappa^{c}\right)|\nabla \varphi|=0$

where $V_{0}$ is the initial total volume of the bubble and $V$ is the total mass corresponding to the level-set function $\varphi(\tau), \kappa$ is the surface curvature. The parameters $a, b$ and $c$ are chosen in the present study as 1,0 and 0 , respectively. In the present study, the change of bubble volume was no more than $1 \%$ in all cases.

Thus, based on the level-set function and the continuum interface model, the dimensionless governing equations can be written as

$\nabla \cdot \boldsymbol{u}=0$

$$
\begin{aligned}
& \frac{\partial \boldsymbol{u}}{\partial t}+\boldsymbol{u} \cdot \nabla \boldsymbol{u} \\
& \quad=-\frac{1}{\rho^{\prime}}\left\{\begin{array}{l}
\nabla p+\frac{\mu^{\prime} \nabla \cdot\left(\nabla \boldsymbol{u}+\nabla^{T} \boldsymbol{u}\right)}{R e} \\
+\left[\frac{1-C a\left(T-T_{0}\right)}{W e} \kappa \boldsymbol{n}+\frac{(\mathbf{I}-\boldsymbol{n n}) \cdot \nabla T}{R e}\right] \delta(\varphi)
\end{array}\right\}
\end{aligned}
$$

$$
\rho^{\prime} c_{p}^{\prime}\left(\frac{\partial T}{\partial t}+\boldsymbol{u} \cdot \nabla T\right)=\frac{k^{\prime}}{M a} \nabla^{2} T
$$

in which $R, U$, and $\left|\nabla T_{\infty}\right| R$ are used as the characteristic length, velocity and temperature, respectively. $T_{0}$ is a dimensionless reference temperature which is set as 0 , or the value at the initial position of the center of the bubble in the present study. The other dimensionless parameters appeared in the above equations are defined as follows

$\rho^{\prime}=\frac{\rho}{\rho_{1}}, \mu^{\prime}=\frac{\mu}{\mu_{1}}, c_{p}{ }^{\prime}=\frac{c_{p}}{c_{p 1}}$,

$k^{\prime}=\frac{k}{k_{1}}, C a=\frac{\mu U}{\sigma_{0}}, W e=\operatorname{Re} C a$.

The last two dimensionless parameters are the capillary and Weber numbers, which determine the deformation of the bubble.

The unit surface normal, the surface curvature and the delta function appeared in the above equations are also defined as follows

$\boldsymbol{n}=\nabla \varphi /|\nabla \varphi|$

$\kappa=\nabla \cdot \boldsymbol{n}$

$\delta(\varphi)=\left\{\begin{array}{cr}1+\cos \left(\frac{2 \pi \varphi}{3 h}\right) /(3 h) & (|\varphi|<1.5 h) \\ 0 & (|\varphi| \geq 1.5 h)\end{array}\right.$

where $h$ is the grid spacing.

Furthermore, in order to avoid numerical instability caused by their jumps across the interface, the material properties of the fluids are smoothed by the Heaviside function

$\varsigma=\varsigma_{2}+\left(\varsigma_{1}-\varsigma_{2}\right) H$

where $\varsigma$ represents $\rho, \mu$, and so on. The Heaviside function $H$ is defined as

$H= \begin{cases}1 \\ \frac{1}{2}+\frac{\varphi}{3 h}+\sin \left(\frac{2 \pi \varphi}{3 h}\right) / 2 \pi & (|\varphi|<1.5 h) \\ 0 & (\varphi \leq-1.5 h)\end{cases}$

The projection method is used to solve the above governing equations with uniform staggered grid of equal spacing at the following initial conditions

$u=v=0, \quad T=z(t=0)$ 
and boundary conditions:

$$
\left\{\begin{array}{lll}
u=v=0, & T=-A & (z=-A) \\
u=v=0, & T=B & (z=B) \\
u=\frac{\partial v}{\partial r}=\frac{\partial T}{\partial r}=0 & & (r=0) \\
u=v=0, & T=z & (r=C)
\end{array}\right.
$$

where $z=-A, z=B$, and $r=C$ denote the bottom, top, and the outer boundaries, respectively. The center of the bubble locates at the point $(0,0)$ at the beginning, and $A$ is always set as 3 .

\section{Resolution Test and Validation}

In order to estimate the accuracy of the numerical algorithm, the method described above is first applied to several test cases. The dimensionless numbers except $R e$ and $M a$ are chosen as $\xi=\rho_{2} / \rho_{1}=$ $1 / 1000, \alpha=\mu_{2} / \mu_{1}=1 / 1000, \beta=k_{2} / k_{1}=1 / 30, \chi=$ $c_{p 2} / c_{p 1}=1 / 4$, and $C a=0.2$.

Figure 1 shows the comparison between the numerical result for a test case of $R e=0.01$ and $M a=$ 0.01 and the analytical prediction of YGB theory. A computational domain of $15 \times 5$ and a grid of $300 \times$ 100 are used. A good agreement is evident since the effects of convective transport of momentum and heat are negligible in this case.

Before the above validation, the influence of the grid spacing is firstly studied at $R e=1$ and $M a=100$ with a computational domain of $15 \times 5$, namely $A=3, B=12$, and $C=5$. It is found that the difference of the terminal migration velocity is no more than $1 \%$ between two grids of $300 \times 100$ and $450 \times 150$. The influence of the computational domain is also studied with the same grid spacing corresponding to the first one of the above cases. It is also found that differences of the terminal

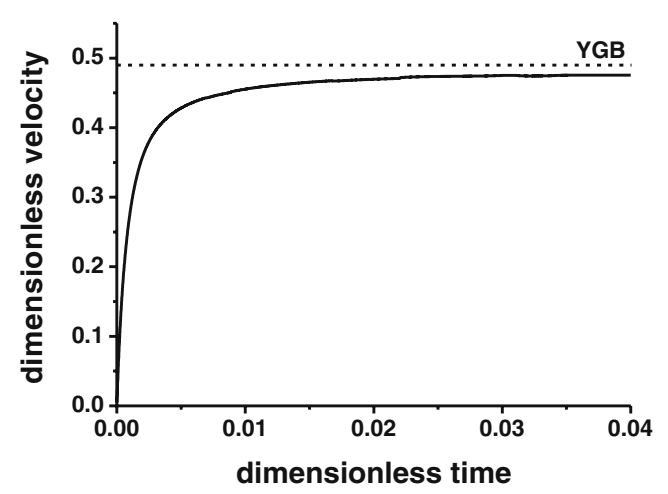

Fig. 1 Comparison between the numerical result and the YGB theory migration velocity are no more than $1 \%$ among three computational domains of $15 \times 5,20 \times 5$, and $15 \times$ 7. Thus, all results reported in the present paper are obtained with the computational domain of $15 \times 5$ and the grid of $300 \times 100$.

\section{Influence of Dimensionless Parameters}

Figure 2 presents evolutions of bubble migration velocity for different Marangoni number at a fixed Reynolds number of 1 . The other dimensionless parameters are the same as those in Fig. 1. The early transients of the motion, caused by the preternatural initial conditions used in the numerical simulations, show the same characteristics as those reported by Oliver and De Witt (1994) and Welch (1998). Steady state of the thermocapillary migration can be reached, which we mainly concerned with. It is evident that the migration velocity is a monotonically decreasing function of the Marangoni number, which is consistent with the previous theoretical and numerical studies for the case of nondeformable gas bubbles.

The dependence of the migration velocity on the Marangoni number can easily be explained by the isotherms surrounding the bubble, which are shown in Fig. 3 equally-spaced with dimensionless temperature increments of 0.4. Obviously, the enhanced convective transport of momentum and heat with the increase of the Marangoni number results in the wrapping of the isotherms around the front of the bubble, leading to a substantial reduction in the surface temperature gradient and diminishing the driving force for the motion of the bubble over much of the front surface.

In theoretical and numerical studies of the thermocapillary migration of gas bubbles, the influence of the gas phase inside the bubble are usually ignored, and

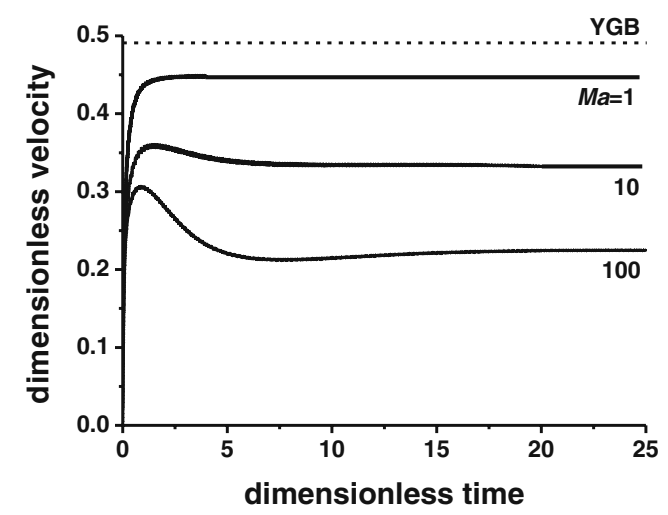

Fig. 2 Influence of Marangoni number on evolution of bubble migration velocity 
Fig. 3 The temperature fields at different Marangoni number. a $M a=1$ b $M a=10, \mathbf{c} M a=100$

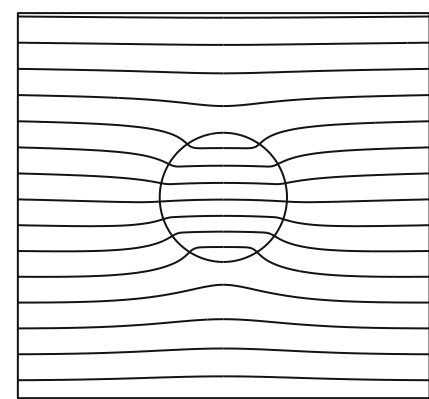

A) $M a=1$

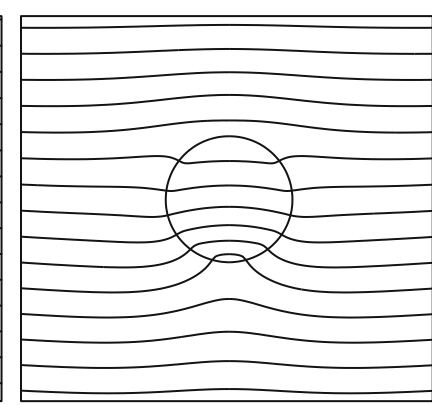

B) $M a=10$

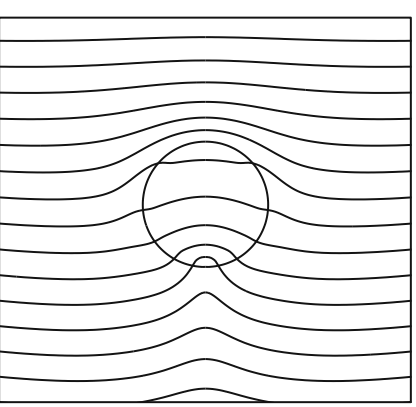

C) $M a=100$ then the limits of $\alpha=0$ and $\beta=0$ are assumed. The finite values, however, of the material parameters of real gas may have some influence on the thermocapillary migration of gas bubble. Figures 4 and 5 show respectively the influences of the viscosity ratio $\alpha$ and the thermal conductivity ratio $\beta$ on the terminal migration velocity at $R e=1$ and $M a=100$. The other parameters are the same as those in Fig. 1.

In these figures, the terminal migration velocities $V$ of bubbles are scaled using the corresponding values predicted by the YGB theory. Thus, according to Eq. 1 , the scaled velocities are equal to 1 for any values of $\alpha$ and $\beta$ in the absence of convective transport of momentum and heat. On the contrary, the results shown in Figs. 4 and 5 indicate a different trend in which the scaled velocity decreases with the decrease of $\alpha$ or $\beta$. Furthermore, the scaled velocity may keep a nearly constant value if $\alpha<0.1$, while a monotone decrease of the scaled velocity can be observed with the decrease of $\beta$ throughout its possible range. Thus, the influence of the convective transport of heat inside bubbles cannot be neglected, while the influence of the

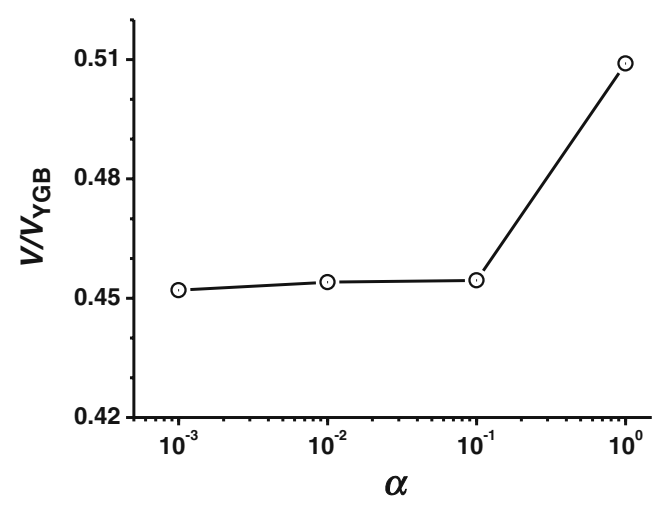

Fig. 4 The scaled migration velocity as a function of dynamic viscosity ratio convective transport of momentum inside bubbles may be actually negligible.

\section{Comparison with Space Experimental Data}

The thermocapillary motion of deformable air bubbles in silicone oil is studied numerically using the above algorithm at the same conditions as those in the space experiments of Hadland et al. (1999) and Kang et al. (2008). Constant material properties, however, are assumed to be independent of temperature. The following values are adopted for the dimensionless parameters in the problem, namely $\xi=0.0013, \alpha=0.0035$, $\beta=0.21, \chi=0.63$, and $\operatorname{Pr}=83.3$. Furthermore, a much larger value for the capillary number, i.e. $C a=0.2$ which is still much less than 1 to guarantee no distinct deformation of the bubble, is used here than those in the space experiments, which are of the order of $10^{-2}$ or less, in order to prevent the virtual flow caused by the strong jump of the normal stress across the interface of bubbles.

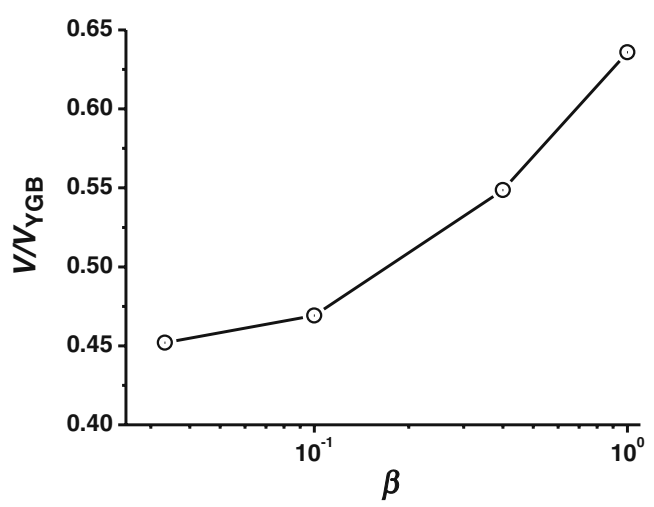

Fig. 5 The scaled migration velocity as a function of thermal conductivity ratio 


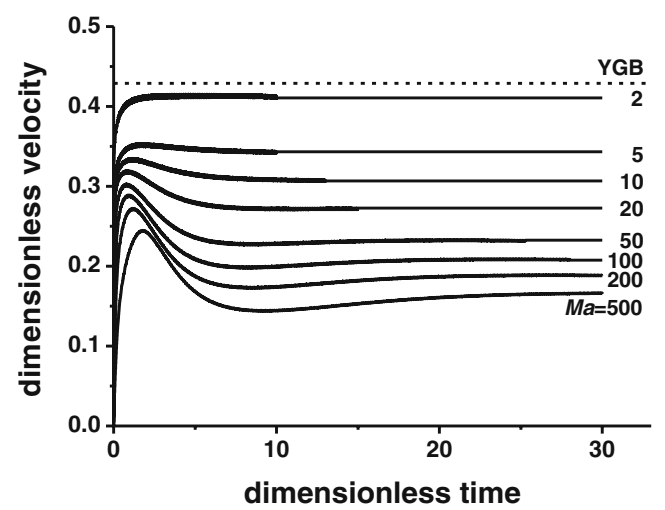

Fig. 6 Evolutions of bubble migration velocity at different Marangoni number

Figure 6 shows evolutions of bubble migration velocities at different Marangoni number. A steady state can always be reached for the thermocapillary migration of air bubbles. The predicted dimensionless terminal velocity is a monotonically decreasing function of the Marangoni number.

In Fig. 7, the predicted terminal migration velocities, which are also scaled using the corresponding values predicted by the YGB theory, are compared with the experimental data of Hadland et al. (1999) and Kang et al. (2008), labeled respectively as HBWS1999 and KCHD-2008 for the brevity of the figure. Generally, good agreements are evident. Asymptotic analysis of Shankar and Subramanian (1988), SS1988, for small Marangoni number case and those of Balasubramaniam and Subramanian (1996), BS-1996, for large Marangoni number case are also plotted in Fig. 7a for comparison, while numerical predictions of Shankar and Subramanian (1988), Balasubramaniam and Lavery (1989), and Ma (1998), labeled respectively as SS-1988, BS-1989 and Ma-1998 are plotted in Fig. 7b.

In Fig. 7b, a nearly perfect agreement of the present results with those of Shankar and Subramanian (1988) should be considered as an incidental event, since the influences of convective and molecular transports of momentum and heat inside bubbles were ignored and an undeformable bubble with a fixed spherical shape were assumed in the latter. The scaled velocity will increase if the influences of transports inside bubbles are taken into account because of the decrease of the YGB velocity. Due to the same reason, the differences between the present results and those of Balasubramaniam and Lavery Balasubramaniam and Lavery (1989) or Ma (1998) may be slightly enlarged. On the other hand, a higher value of the Prandtl number within the actual range in space experiments is adopted here, which can lead to an underestimated value of the Reynolds number. Furthermore, as mentioned above, a much larger value of the capillary number is also adopted here than that in space experiments. According to the findings by Haj-Hariri et al. (1997) and Yin et al. (2008) in their numerical studies of the thermocapillary migration of liquid drops with or without deformation, the terminal migration velocities may increase with the increase of the Reynolds number, particularly at high Marangoni number, and may decrease with the increase of the capillary number. Thus, the differences may be diminished if these influences are taken into account.

Isotherms in the terminal steady state of the thermocapillary migration for several typical cases are shown as dashed lines in Fig. 8 in a meridian plane. Streamlines are also plotted as solid lines in the same figure, in which the left part is those in the local reference frame attached to the center of the bubble and the right part is those in the laboratory reference frame. As mentioned above, the enhanced convective transport of momentum and heat with the increase of the Marangoni number leads to a substantial reduction in the surface
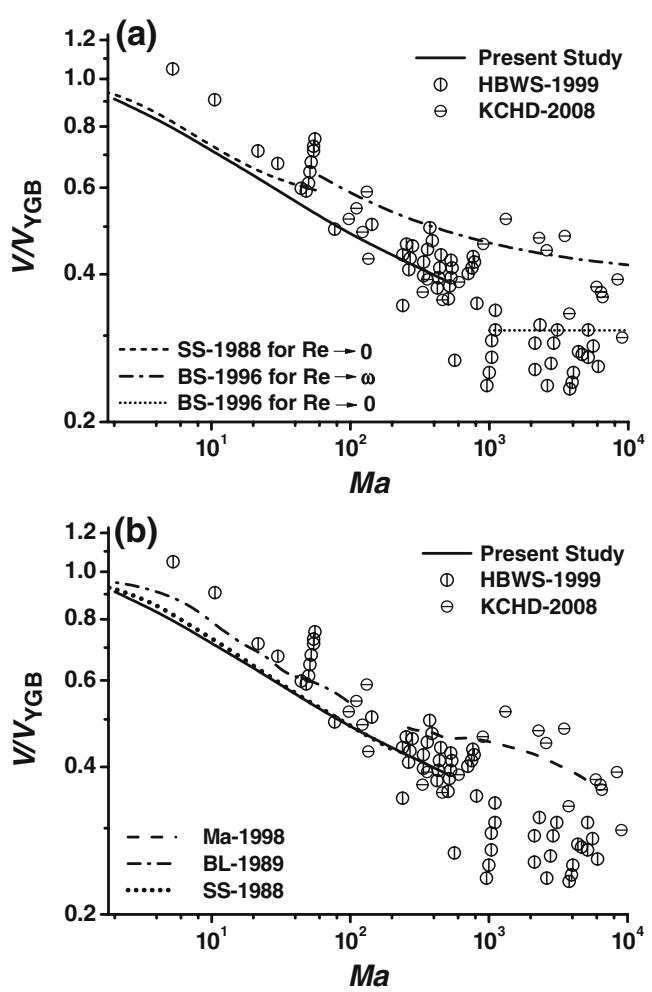

Fig. 7 Comparison of the present numerical predictions of the terminal thermocapillary migration velocity with experimental data and (a) asymptotic predictions, (b) other numerical predictions 
Fig. 8 Isotherms (dashed line) and streamlines (solid line) at different Marangoni number

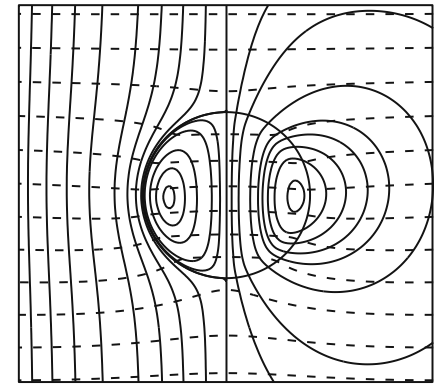

A) $\mathrm{Ma}=2$

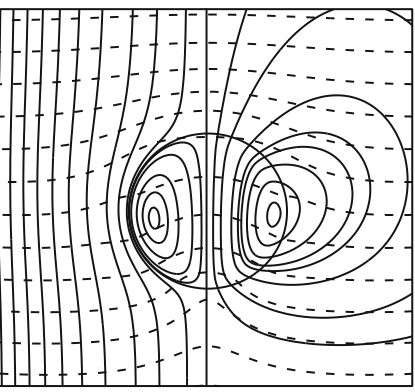

B) $\mathrm{Ma}=50$

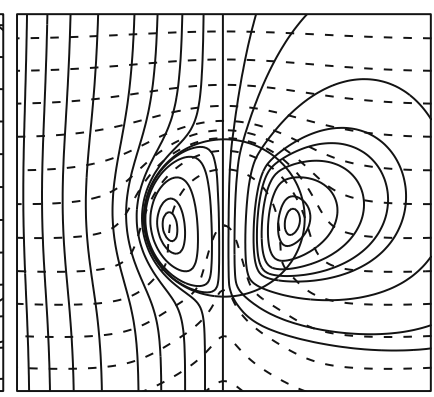

C) $\mathrm{Ma}=500$ temperature gradient and diminishes the driving force for the motion of the bubble over much of the front surface. That is the reason of the fact that the migration velocity decreases monotonically with the increase of the Marangoni number.

An annular vortex can be observed inside the bubble. The center of the vortex locates near the interface of the bubble. As shown in Table 1, the transverse position of the vortex is not changed with the Marangoni number, while its longitudinal position moves downstream with the increase of the Marangoni number. The reason for this fact should be found in the distribution of temperature. According to Fig. 8, the temperature gradient is nearly uniform along the bubble interface at small Marangoni number, while a much uneven distribution of temperature will be observed at high Marangoni number. Although the surface temperature gradient over whole surface decreases at high Marangoni number, it becomes larger along the rear surface than that along the front surface. Larger temperature gradient means larger driving force and faster motion, so the center of the vortex inside bubbles

Table 1 The locations of the center of the vortex inside bubbles in the local reference frame attached to the center of bubbles (I) and in the laboratory reference frame (II)

\begin{tabular}{lll}
\hline$M a$ & $(r, z)_{\mathrm{I}}$ & $(r, z)_{\mathrm{II}}$ \\
\hline 2 & $(0.68,-0.0312)$ & $(0.84,-0.0112)$ \\
5 & $(0.68,-0.0563)$ & $(0.84,-0.0163)$ \\
10 & $(0.68,-0.0699)$ & $(0.84,-0.0299)$ \\
20 & $(0.68,-0.0740)$ & $(0.84,-0.0240)$ \\
50 & $(0.68,-0.0802)$ & $(0.84,-0.0402)$ \\
100 & $(0.68,-0.0948)$ & $(0.84,-0.0548)$ \\
200 & $(0.68,-0.0958)$ & $(0.84,-0.0552)$ \\
500 & $(0.68,-0.0968)$ & $(0.84,-0.0568)$ \\
\hline
\end{tabular}

The origins of the two frames are located at the center of bubbles moves downstream with the increase of the Marangoni number.

Figure 9 shows the aspect ratio between the longitudinal and transverse lengths of deformed bubbles in the terminal steady state of the thermocapillary migration. It decreases from 1 quickly with the increase of the Marangoni number at $M a<100$, while the decreasing rate lowers when the Marangoni number exceeds 100. The aspect ratio smaller than 1 indicates the bubble becomes a slightly oblate sphere, which is consistent with Welch (1998). The biggest variation of aspect ratio, however, is no more than $2 \%$ in the present study. It ought to be pointed out here that the actual deformation may be much smaller than the predictions since the capillary number in space experiments has much smaller values than that used here. Therefore, no distinct influence of the deformation of bubbles can occur in the thermocapillary migration of gas bubbles, at least within the parameter ranges studied by Hadland et al. (1999) and Kang et al. (2008).

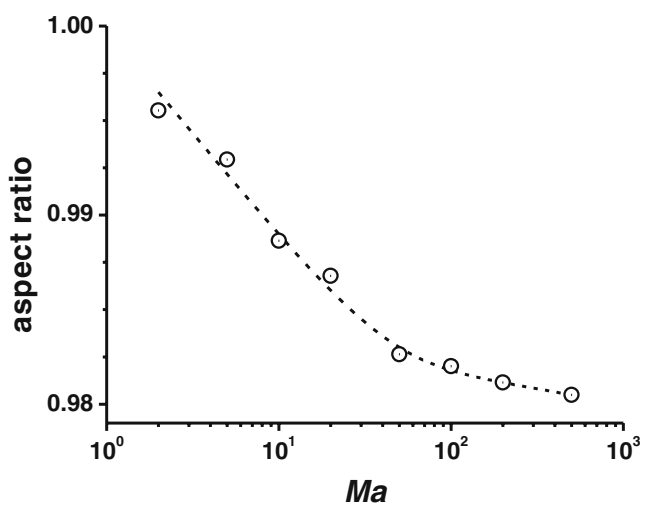

Fig. 9 Aspect ratio of the deformable air bubble at different Marangoni number 


\section{Conclusions}

The thermocapillary migration of deformable gas bubbles has been studied numerically at moderate to large Marangoni number. The axisymmetric governing equations are solved using the projection method with a uniform staggered grid of equal spacing at appropriate initial and boundary conditions. The level-set method and the continuum interface model are used to account for finite bubble deformation.

It is found from the numerical results that steady state of the thermocapillary migration of deformable gas bubbles can always be reached. The terminal migration velocity of bubbles scaled by the named YGB velocity decreases with the decrease of the ratios of the dynamic viscosity and thermal conductivity between the gas and continuous bulk liquid phases. The scaled velocity may keep a nearly constant value if the ratio of the dynamic viscosity is less than 0.1 , while a monotone decrease of the scaled velocity can be observed with the decrease of the ratio of the thermal conductivity throughout its possible range. It is suggested that the influence of the convective transport of heat inside bubbles cannot be neglected, while the influence of the convective transport of momentum inside bubbles may be actually negligible.

The numerical results of the simulations under the conditions corresponding to the space experiments of Hadland et al. (1999) and Kang et al. (2008) are compared with experimental data, asymptotic predictions and other numerical simulations. Good agreement can be observed. The structures of the flow and temperature fields are also presented to reveal the influences of convective transport on the phenomenon. It is verified by the present results that no distinct influence of the deformation of bubbles can occur in the thermocapillary migration of gas bubbles, at least within the parameter range studied by Hadland et al. (1999) and Kang et al. (2008).

Acknowledgement The present work is supported financially by the National Natural Science Foundation of China under the grant of 10972225 .

\section{References}

Balasubramaniam, R., Lavery, J.E.: Numerical simulation of thermocapillary bubble migration under microgravity for large Reynolds and Marangoni numbers. Numer. Heat Transf. A 16(2), 175-187 (1989)

Balasubramaniam, R., Subramanian, R.S.: Thermocapillary bubbles migration-thermal boundary layers for large Marangoni numbers. Int. J. Multiph. Flow 22(3), 593-612 (1996)
Bratukhin, Y.K.: Termokapillyarnyy dreyf kapel'ki vyazkoy zhidkosti. Izv. Akad. Nauk SSSR, Meh. Zhidk. Gaza 5, 156-161 (1975)

Chen, J.C., Lee, Y.T.: Effect of surface deformation on thermocapillary bubble migration. AIAA J. 30(4), 993-998 (1992)

Crespo, A., Jimenez-Fernandez, J.: Thermocapillary migration of bubbles at moderately large Reynolds numbers. In: Rath, H.J. (ed.) Microgravity Fluid Mechanics IUTAM Symposium, Bremen 1991, pp. 405-411. Springer, Berlin (1992a)

Crespo, A., Jimenez-Fernandez, J.: Thermocapillary migration of bubbles: a semi-analytical solution for large Marangoni numbers. In: Proceedings of the 8th European Symposium on Materials Fluid Science (ESA SP-333), 12-16 April 1992, pp. 193-196. Brussels, Belgium (1992b)

Crespo, A., Migoya, E., Manuel, E.: Thermocapillary migration of bubbles at large Reynolds number. Int. J. Multiph. Flow 24(4), 685-692 (1998)

Hadland, P.H., Balasubramaniam, R., Wozniak, G., Subramanian, R.S.: Thermocapillary migration of bubbles and drops at moderate to large Marangoni number and moderate Reynolds number in reduced gravity. Exp. Fluids 26, 240-248 (1999)

Haj-Hariri, H., Shi, Q., Borhan, A.: Thermocapillary motion of deformable drops at finite Reynolds and Marangoni numbers. Phys. Fluids 9(4), 845-855 (1997)

Kang, Q., Cui, H.L., Hu, L., Duan, L.: On-board experimental study of bubble thermocapillary migration in a recoverable satellite. Microgravity Sci. Technol. 20(2), 67-71 (2008)

Ma, X.J.: Numerical simulation and experiments on liquid drops in a vertical temperature gradient in a liquid of nearly the same density. PhD thesis, Clarkson University, Potsdam, New York, USA (1998)

Merritt, R.M.: Bubble migration and interactions in a vertical temperature gradient. $\mathrm{PhD}$ thesis, Clarkson University, Potsdam (1988)

Nas, S., Tryggvason, G.: Computational investigation of the thermal migration of bubbles and drops. In: Proceedings of th eASME Winter Annual Meeting (AMD-174/FED-175), pp. 71-83 (1993)

Oliver, D.L.R., De Witt, K.J.: Transient motion of a gas bubble in a thermal gradient in low gravity. J. Colloid Interface Sci. 164, 263-268 (1994)

Osher, S., Sethian, J.A.: Fronts propagating with curvaturedependent speed: algorithms based on Hamilton-Jacobi formulations. J. Comput. Phys. 79(1), 12-49 (1988)

Shankar, N., Subramanian, R.S.: The stokes motion of a gas bubble due to interfacial tension gradients at low to moderate Marangoni numbers. J. Colloid Interface Sci. 123(2), 512$522(1988)$

Son, G., Dhir, V.K.: A level set method for analysis of film boiling on an immersed solid surface. Numer. Heat Transf. B 52(12), 153-177 (2007)

Subramanian, R.S.: Slow migration of a gas bubble in a thermal gradient. AIChE J 27(4), 646-654 (1981)

Subramanian, R.S.: Thermocapillary migration of bubbles and droplets. Adv. Space Res. 3(5), 145-153 (1983)

Subramanian, R.S., Balasubramaniam, R.: The Motion of Bubbles and Drops in Reduced Gravity. Cambridge University Press, Cambridge (2001)

Sussman, M., Smereka, P., Osher, S.: A level set approach for computing solutions to incompressible two-phase flow. J. Comput. Phys. 114(1), 146-159 (1994)

Szymczyk, J.A., Siekmann, J.: Numerical calculation of the thermocapillary motion of a bubble under microgravity. Chem. Eng. Commun. 69(1), 129-147 (1988) 
Thompson, R.L., DeWitt, K.J., Labus, T.L.: Marangoni bubble motion phenomenon in zero gravity. Chem. Eng. Commun. 5, 299-314 (1980)

Treuner, M., Galindo, V., Gerbeth, G., Langbein, D., Rath, H.J.: Thermocapillary bubble migration at high Reynolds and Marangoni numbers under low gravity. J. Colloid Interface Sci. 179, 114-127 (1996)
Welch, S.W.J.: Transient thermocapillary migration of deformable bubbles. J. Colloid Interface Sci. 208, 500-508 (1998)

Yin, Z., Gao, P., Hu, W., Chang, L.: Thermocapillary migration of nondeformable drops. Phys. Fluids 20, 082101 (2008)

Young, N.O., Goldstein, J.S., Block, M.J.: The motion of bubbles in a vertical temperature gradient. J. Fluid Mech. 6, 350-356 (1959) 